\title{
Dark matter pair associated with a $W$ boson production at the LHC in next-to-leading order QCD
}

\author{
Song Mao, ${ }^{a}$ Li Gang, ${ }^{a}$ Ma Wen-Gan, ${ }^{b}$ Zhang Ren-You ${ }^{b}$ and Guo Jian-You ${ }^{a}$ \\ ${ }^{a}$ School of Physics and Material Science, Anhui University, \\ Hefei, Anhui 230039, P.R. China \\ ${ }^{b}$ Department of Modern Physics, University of Science and Technology of China, \\ Hefei, Anhui 230026, P.R. China \\ E-mail: songmao@mail.ustc.edu.cn, lig2008@mail.ustc.edu.cn, \\ mawg@ustc.edu.cn, zhangry@ustc.edu.cn, jianyou@ahu.edu.cn
}

ABSTRACT: We investigate the QCD next-to-leading order (NLO) corrections to the production of a pair of fermionic dark matter particles associated with a $\mathrm{W}$ boson production through a mediator which couples to standard model particles via either a vector or axialvector coupling at the LHC. We find that the QCD NLO corrections reduce the dependence of the total cross sections on the factorization and renormalization scales, and the $K$-factors increase with the increment of the dark matter mass. We also provide the LO and QCD NLO corrected distributions of the transverse momenta $p_{T}^{\mu}$ of final muon and transverse mass $M_{T}$. We find that the LO cross section is significantly changed by the QCD NLO corrections.

Keywords: QCD Phenomenology, NLO Computations

ARXIV EPRINT: 1403.2142 


\section{Contents}

1 Introduction 1

2 Calculation descriptions $\quad 2$

3 Numerical results and discussion 4

$\begin{array}{llr}4 & \text { Summary } & 8\end{array}$

\section{Introduction}

Observational evidence has confirmed that there exist some kinds of cold non-baryonic dark matter (DM) in our universe, which is the dominant component of cosmical matter [1]. Due to the characteristics of non-baryonic, the dark matter cannot be composed of anyone of known substances in our earth and galaxies. Astrophysical observations also can not tell us about the property of the dark matter particle or whether it interacts with the Standard Model (SM) particles beyond gravitation. Revealing the distribution and nature of dark matter is one of the most interesting current challenges in the fields of both cosmology and particle physics.

Among all the dark matter candidates, weakly interacting massive particle (WIMP) is one of the most compelling versions. Primarily this is due to that it offers a possibility to understand the relic abundance of dark matter as a natural consequence of the thermal history of the universe [2,3]. Some extensions of the SM, such as Supersymmetry [4, 5], Universal Extra Dimensions [6] or Little Higgs Models [7], naturally lead to good candidates for WIMPs and the cosmological requirements for the WIMP abundance in the universe. In these theoretical frameworks, the WIMP candidates are often theoretically well motivated and compelling. However, all of these theories still lack experimental support, and we can not judge which theory is proper for dark matter. Additionally, the first observations of dark matter may come from direct- or indirect-detection experiments, which may not provide information about the general properties of the dark matter particle without offering a way to distinguish between underlying theories. Thus, model independent studies of dark matter phenomenology using effective field theory is particularly important.

Recently, the observed results favor a light DM with a mass around $10 \mathrm{GeV}$ in various experiments, such as DAMA, CoGeNT and XENON10/100 [8, 9, 11, 12]. However, it is difficult to probe in the low-mass region or to constrain the parameter space with direct detection experiments, since the typical energy transfer in the scattering of such particles is small compared to the experimental energy thresholds, and the interpretation of experimental results are affected by astrophysical uncertainties. As colliders are most effective 
when producing highly boosted light WIMPs, one can directly probe the interactions between DM particles and individual SM particles at the LHC. In the case of a WIMP, stability on the order of the lifetime of the universe implies that pair production must highly dominate over single production, and precludes the WIMP from decaying within the detector volume. Therefore, WIMPs appear as missing energy, and can potentially be observed by searching for visible particles recoiling against dark matter particles [13-17]

Searches for dark matter in missing momentum channels can be classified based on the visible particles against which the invisible particles recoil. Existing experimental studies have considered the cases in which the visible radiation is a jet of hadrons (initiated by a quark or gluon) [18-20], a photon [21, 22], or a $W / Z$ boson decaying into leptons or hadronic jets [23-25]. Because the LHC is a proton-proton collider, the QCD correction should be considered for any process if people want to make a reliable prediction. More recently, The production of DM pairs plus a jet or photon have been calculated to QCD next-to-leading order (NLO) [26-30]. However, the production of DM pairs in association with a mono- $W / Z$ has only been considered to LO [31-33]. In this work, using model independent method we investigate the possibility of discovering the DM production in associated with a $W$ boson induced by a dimension six effective operator in the next-toleading order (NLO) QCD.

The paper is organized as follows. In section 2, we briefly describe the related effective field theory and present the details of calculation strategy. In section 3, we give the numerical results and discussion for the process $p p \rightarrow \bar{\chi} \chi+W^{ \pm}+X$. Finally, a short summary is given in section 4 .

\section{Calculation descriptions}

We assume that the dark matter candidate is the only new particle which is singlet under the SM local symmetries, and all SM particles are singlets under the dark-sector symmetries. The interaction between the SM and DM sectors is presumably effected by the exchange of some heavy mediators whose nature we do not need to specify, but only assume that they are much heavier than the typical scales. The effective field theories for dark matter interacting primarily with SM quarks have been considered in refs. [14-17, 34-39]. The most prominent coupling characters are:

$$
\begin{aligned}
\text { Spin - independent vector coupling : (V) } & \frac{1}{\Lambda_{\mathrm{D} 5}^{2}}\left(\bar{\chi} \gamma^{\mu} \chi\right)\left(\bar{q} \gamma_{\mu} q\right) \\
\text { Spin - dependent axial - vector coupling : (AV) } & \frac{1}{\Lambda_{\mathrm{D} 8}^{2}}\left(\bar{\chi} \gamma^{\mu} \gamma^{5} \chi\right)\left(\bar{q} \gamma_{\mu} \gamma^{5} q\right),
\end{aligned}
$$

where $\chi$ is the dark matter particle, which we assume to be a Dirac fermion, $q$ is a SM quark, and the characterizing parameters of the model are the scales of the effective interactions $\Lambda_{i}=\frac{M_{\text {messenger }}}{\sqrt{g_{\chi} g_{q}}}$ between the two sectors. We will typically consider only one interaction type to dominate at a time, and will thus keep one $\Lambda$ being finite while the rest is set to be infinity and decoupled.

There are two different topological diagrams for process $p p \rightarrow \bar{\chi} \chi+W^{ \pm}+X$ at the LO, in which dark matter pair of WIMPs $(\bar{\chi} \chi)$ produced via an unknown intermediate 

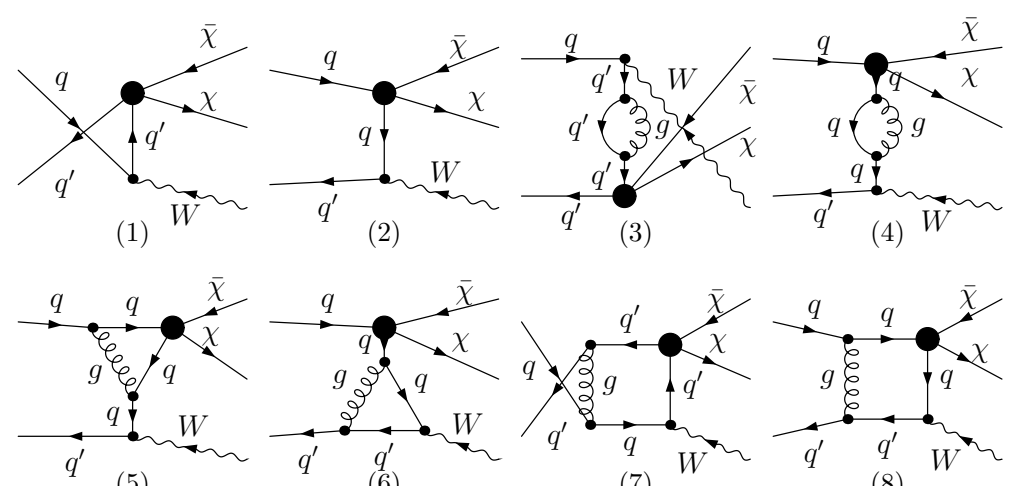

(5)

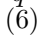

(7)

(8)

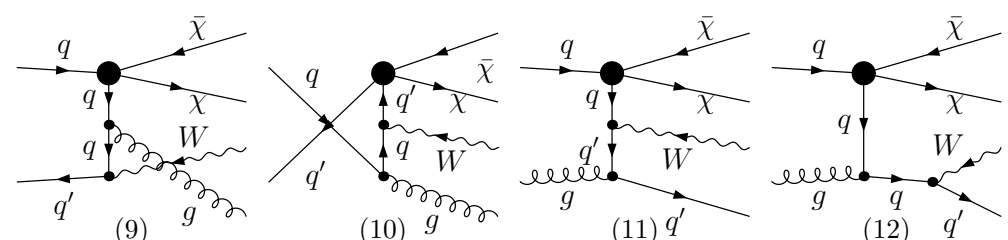

(9)

(10) $\mathrm{g}$

(11) $q^{\prime}$

(12) $q^{\prime}$

Figure 1. Some representative Feynman diagrams for the process $p p \rightarrow \bar{\chi} \chi+W^{ \pm}+X$ at the LO and QCD NLO.

state, with initial state radiation of a $W$ boson. Due to the $C P$-conservation, the cross section for the $q q^{\prime} \rightarrow \bar{\chi} \chi+W^{-}\left(q q^{\prime}=\bar{u} d, \bar{u} s, \bar{c} d, \bar{c} s\right)$ subprocess should be the same as that the corresponding charge conjugate subprocess $q q^{\prime} \rightarrow \bar{\chi} \chi+W^{+}\left(q q^{\prime}=u \bar{d}, u \bar{s}, c \bar{d}, c \bar{s}\right)$ at the parton level. By taking the Cabibbo-Kobayashi-Maskawa (CKM) matrix elements $V_{t d}=V_{t s}=V_{u b}=V_{c b}=0$, the LO contribution to the cross section for the parent process $p p \rightarrow \bar{\chi} \chi+W^{ \pm}+X$ comes from the following subprocesses. We denote the subprocesses as:

$$
\begin{array}{llrl}
q\left(p_{1}\right)+q^{\prime}\left(p_{2}\right) \rightarrow \bar{\chi}\left(p_{3}\right)+\chi\left(p_{4}\right)+W^{+}\left(p_{5}\right), & & \left(q q^{\prime}=u \bar{d}, u \bar{s}, c \bar{d}, c \bar{s}\right) \\
q\left(p_{1}\right)+q^{\prime}\left(p_{2}\right) \rightarrow \bar{\chi}\left(p_{3}\right)+\chi\left(p_{4}\right)+W^{-}\left(p_{5}\right), & & \left(q q^{\prime}=\bar{u} d, \bar{u} s, \bar{c} d, \bar{c} s\right)
\end{array}
$$

where $p_{1}, p_{2}$ and $p_{3}, p_{4}, p_{5}$ represent the four-momenta of the incoming partons and the outgoing dark matter particle $\chi$, and $W^{ \pm}$boson, respectively.

In the QCD NLO calculations, the parent process $p p \rightarrow \bar{\chi} \chi+W^{ \pm}+X$ involves four contribution components: (1) The QCD one-loop virtual corrections to the partonic process $q q^{\prime} \rightarrow \bar{\chi} \chi+W^{ \pm} ;(2)$ The real gluon emission partonic process $q q^{\prime} \rightarrow \bar{\chi} \chi+W^{ \pm}+g ;$ (3) The real light-(anti)quark emission partonic process $q g \rightarrow \bar{\chi} \chi+W^{ \pm}+q^{\prime}$; (4) The corresponding contributions of the parton distribution functions (PDF) counterterms.

Some representative QCD NLO Feynman diagrams for the process $p p \rightarrow \bar{\chi} \chi W^{ \pm}+X$ are shown in figure 1 . In virtual correction calculations, we adopt the definitions of scalar and tensor one-loop integral functions in refs. [40, 41]. Using the Passarino-Veltman (PV) method [40, 42], the tensor integrals are expressed as a linear combination of tensor structures and coefficients, where the tensor structures depend on the external momenta and the metric tensors, and the coefficients depend on scalar integrals, kinematics invariants and the dimension of the integral. After the tensor integral reduction is performed, the fundamental building blocks are one-loop scalar integrals. we adopt the dimensional regularization (DR) method in $D=4-2 \epsilon$ dimensions to isolate the ultraviolet (UV), soft 
infrared (IR), and collinear IR divergences. The one-loop scalar integrals arise from IR divergent box diagrams with several external and internal massless particles is most difficult. We adopt the expressions in ref. [43] to deal with the IR divergences in Feynman integral functions, and apply the expressions in refs. [44-46] to implement the numerical evaluations for the IR safe parts of N-point integrals. The UV divergences are removed by the counter terms fixed by the on-mass-shell renormalization condition. Nevertheless, it still contains soft/collinear IR singularities. The infrared (IR) singularities from the one-loop integrals need to be cancelled by adding the contributions of the real gluon emissions.

The real gluon emission subprocess $q q^{\prime} \rightarrow \bar{\chi} \chi W^{ \pm} g$ contains both soft and collinear IR singularities which can be conveniently isolated by adopting the two cutoff phase space slicing (TCPSS) method [47]. The soft divergences from real gluon emission will be canceled by similar singularities from the contribution of the one-loop diagrams. The collinear divergences from real gluon emission corrections, part of it can be eliminated by collinear singularities in virtual corrections, and the remaining collinear divergences in real gluon corrections can be absorbed into the PDFs. Then the UV and IR singularities are exactly vanished after combining the renormalized virtual corrections with the contributions of the real gluon emission processes and the PDF counterterms together. These cancelations can be verified numerically in our numerical calculations.

The real light-quark emission subprocesses have the same order contribution with previous real gluon emission subprocess. These subprocesses contain only the initial state collinear singularities. Using the TCPSS method, the phase space is divided collinear region and non-collinear region. The cross section in the non-collinear region is finite and can be evaluated in four dimensions using the general Monte Carlo method. While the collinear singularity in collinear region can be absorbed into the redefinition of the PDFs at the NLO. We have implemented all above calculations in the way as presented in our previous works $[48,49]$.

\section{Numerical results and discussion}

In this section we present the numerical results and discussions for the $p p \rightarrow \bar{\chi} \chi+W^{ \pm}+X$ process at both the LO and the QCD NLO. We take CTEQ6L1 PDFs with an one-loop running $\alpha_{s}$ in the LO calculation and CTEQ6M PDFs with a two-loop $\alpha_{s}$ in the NLO calculation [50], and the corresponding fitted values $\alpha_{s}\left(M_{Z}\right)=0.130$ and $\alpha_{s}\left(M_{Z}\right)=0.118$ are used for the LO and NLO calculations, respectively. For simplicity we define the factorization and the renormalization scale being equal, and take $\mu \equiv \mu_{f}=\mu_{r}=\left(2 m_{\chi}+\right.$ $\left.m_{W}\right) / 2$ by default unless stated otherwise. We adopt all the quark masses $m_{u}=m_{d}=m_{c}=$ $m_{s}=0$ and employ the following numerical values for the relevant input parameters [51],

$$
\alpha\left(m_{Z}\right)^{-1}=127.918, \quad m_{W}=80.398 \mathrm{GeV}, \quad m_{Z}=91.1876 \mathrm{GeV} .
$$

The CKM matrix elements are fixed as

$$
V_{\mathrm{CKM}}=\left(\begin{array}{lll}
V_{u d} & V_{u s} & V_{u b} \\
V_{c d} & V_{c s} & V_{c b} \\
V_{t d} & V_{t s} & V_{t b}
\end{array}\right)=\left(\begin{array}{ccc}
0.97418 & 0.22577 & 0 \\
-0.22577 & 0.97418 & 0 \\
0 & 0 & 1
\end{array}\right) .
$$




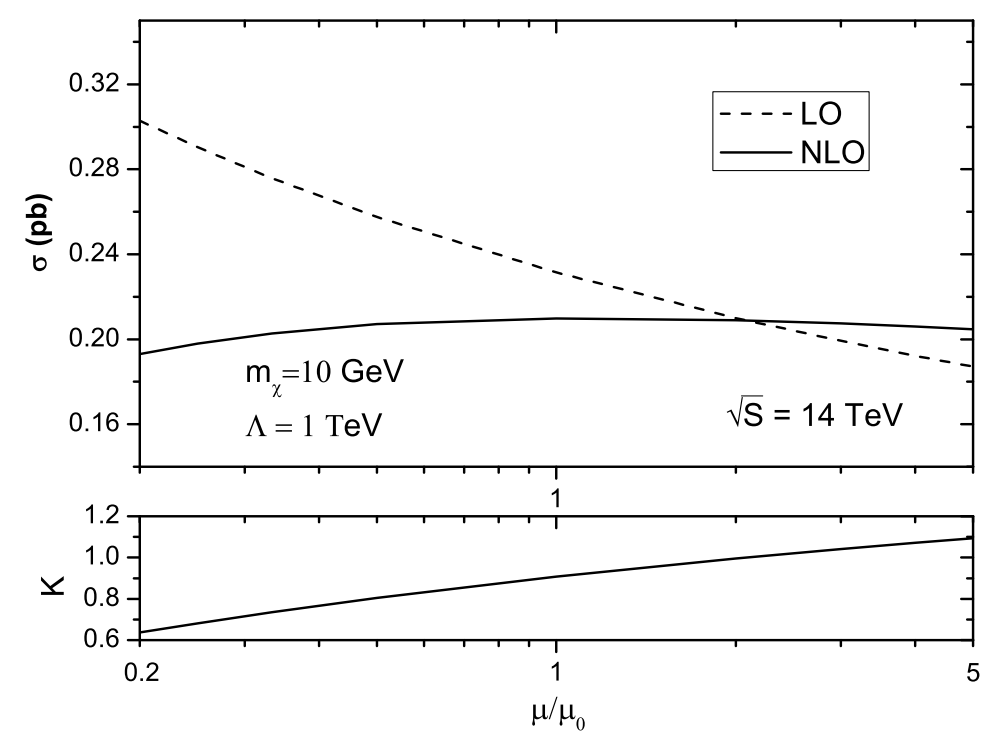

Figure 2. The dependence of the LO, NLO QCD corrected total cross sections and the corresponding $K$-factors $\left(K(\mu) \equiv \sigma_{N L O}(\mu) / \sigma_{L O}(\mu)\right)$ on the factorization/renormalization scale $\left(\mu / \mu_{0}\right)$ for the process $p p \rightarrow \bar{\chi} \chi+W^{ \pm}+X$ at the $\sqrt{s}=14 \mathrm{TeV}$ LHC by taking $m_{\chi}=10 \mathrm{GeV}$ and $\Lambda=1 \mathrm{TeV}$. Here we assume $\mu=\mu_{r}=\mu_{f}$ and define $\mu_{0}=\left(2 m_{\chi}+m_{W}\right) / 2$.

In order to verify the correctness of our tree-level calculation, we compare our numerical results with those in ref. [24] in the same input parameters. We find that they are consistent within the allowable error range. In the calculation of real corrections, we adopt the two-cutoff phase space slicing method [47]. The two phase space cutoffs $\delta_{s}$ and $\delta_{c}$ are chosen as $\delta_{s}=10^{-3}$ and $\delta_{c}=\delta_{s} / 50$ as default choice. In checking the independence of the final results on two cutoffs $\delta_{s}$ and $\delta_{c}$, we find the invariance with the $\delta_{s}$ running from $10^{-2}$ down to $10^{-4}$ within the error control.

In figure 2, we illustrate the renormalization/factorization scale dependence of the LO, NLO QCD corrected total cross sections and the corresponding $K$-factors $(K(\mu) \equiv$ $\left.\sigma_{N L O}(\mu) / \sigma_{L O}(\mu)\right)$ for the process $p p \rightarrow \bar{\chi} \chi+W^{ \pm}+X$. We assume $\mu=\mu_{r}=\mu_{f}$ and define $\mu_{0}=\left(2 m_{\chi}+m_{W}\right) / 2$, where the mass of DM and coefficients $\Lambda$ are taken as $m_{\chi}=$ $10 \mathrm{GeV}$ and $\Lambda=1 \mathrm{TeV}$. Since the difference of theory predicts induced by the vector (D5) operator and axial-vector (D8) is very small, the lines for D5 and D8 in figure 2 look essentially the same that it is difficult to distinguish between them. We can see that the dependence of the NLO cross section on the factorization/renormalization scale are significantly reduced comparing with the LO cross section. This makes the theoretical predictions much more reliable. When the renormalization/factorization scale varies from $0.2 \mu_{0}$ to $5 \mu_{0}$, the corresponding $K$-factor increases from 0.64 to 1.09 for both the vector (D5) and axial-vector (D8) operator.

In figure 3, we show the DM mass dependence of the LO and QCD NLO corrected cross sections for the process $p p \rightarrow \bar{\chi} \chi+W^{ \pm}+X$ with $\Lambda=1 \mathrm{TeV}$ at the $14 \mathrm{TeV}$ LHC induced by 

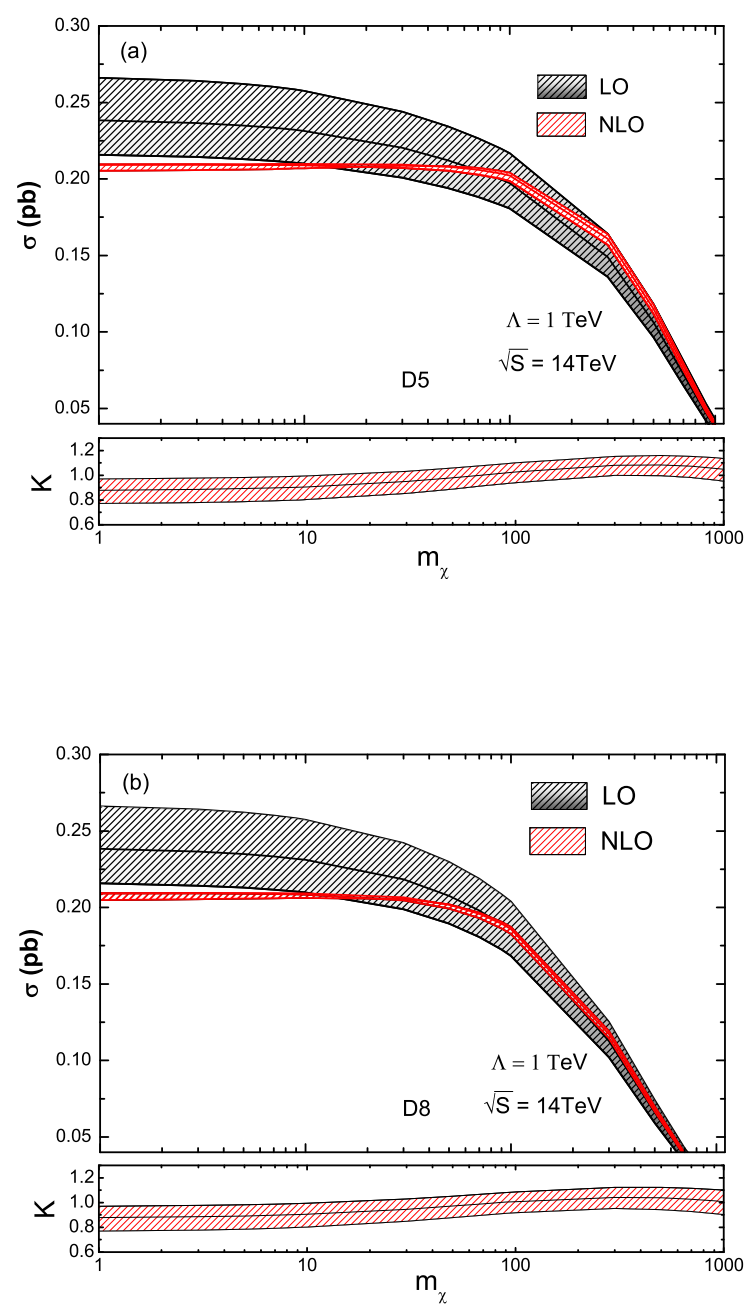

Figure 3. The LO, QCD NLO corrected integrated cross sections and the corresponding $K$-factors as the functions of the DM mass for DM production in association with mono- $W$ boson at the $14 \mathrm{TeV}$ LHC with $\Lambda=1 \mathrm{TeV}$ with the default scale $\mu=\mu_{0}$, the shaded band represents the deviation from this scale when the scales are varied by a factor of two in each direction. (a) for the vector (D5) (b) for the axial-vector (D8).

the vector (D5) and axial-vector (D8) operator. The dependence on the renormalization and factorization scales, taken to be equal $\left(\mu=\mu_{r}=\mu_{f}\right)$, is illustrated by the shaded band linking the predictions obtained at $\mu=2 \mu_{0}$ and $\mu=1 / 2 \mu_{0}$, whilst the central scale choice $\mu=\mu_{0}$ is illustrated by the curve inside the shaded band. When the DM mass varies from $1 \mathrm{GeV}$ to $1000 \mathrm{GeV}$, the QCD NLO corrections modify the LO cross sections obviously. As we expected, the overall scale dependence up to NLO is smaller than the LO prediction. In the DM mass range from 1 to $90 \mathrm{GeV}$, we see that the QCD NLO corrections generally reduce the LO cross sections particularly significant in the lower DM mass range, 
and the $K$-factors increase from 0.88 to 1.0 for both the vector (D5) and axial-vector (D8) operator. Thus, it is necessary to consider the QCD NLO corrections for the process of DM production at hadron colliders. The contributions of the vector (D5) and axial-vector (D8) operator can be distinguished until the DM mass being more than $100 \mathrm{GeV}$. The similar behavior is demonstrated in the monojet production at the LHC [27]. The vector and axial-vector DM operators show similar behaviour in terms of K-factors and scale dependence. This is because that in the massless limit the only terms which are sensitive to the axial nature of the coupling are the four-quark amplitudes, which are a small part of the total NLO cross section. As the the DM mass grows, so does the difference between the operators, with the axial operator being smaller than the vector over the entire mass range.

In measuring the process $p p \rightarrow \bar{\chi} \chi+W^{ \pm}+X$, the finial $\bar{\chi}$ and $\chi$ particles are undetected as the missing energy which will escape the detector without being detected, and the $W$ boson is unstable and will decay to lepton and neutrino. The neutrino is also not detected directly, and gives rise to experimentally missing energy. We investigate the kinematic distributions of final products after the subsequent decays of $W$ gauge boson (i.e., $\left.W^{ \pm} \rightarrow \mu^{ \pm} \stackrel{(-)}{\nu}_{\mu}\right)$. The SM leptonic decay branch ratio of $W$ bosons is employed in further numerical calculations, i.e., $\operatorname{Br}\left(W^{-} \rightarrow \mu^{-} \bar{\nu}_{\mu}\right)=10.57 \%$ [51]. The signature of the $\bar{\chi} \chi W^{ \pm}$production at the LHC including their subsequent decay can be written as

$$
p p \rightarrow \bar{\chi} \chi W^{ \pm}+X \rightarrow \bar{\chi} \chi \mu^{ \pm}{\stackrel{(-)}{\nu_{\mu}}+X}
$$

Then that signal event is detected at the LHC as the detection of one charged lepton $\mu^{ \pm}$ plus missing energy. In analogous to the definition in CMS data analysis [24], we select the events including a muon with $p_{T}^{\mu}>25 \mathrm{GeV}$. The parameter describing DM effect is adopted in terms of the invariant transverse mass, which is defined as

$$
M_{T}=\sqrt{2 p_{T}^{\mu} p_{T}^{\operatorname{miss}}\left(1-\cos \Delta \phi_{\mu D}\right)}
$$

where $p_{T}^{\text {miss }}=E_{T}^{\text {miss }}$ is the missing transverse momentum due to the undetected final DM particle and neutrino, and $\Delta \phi_{\mu D}$ is the azimuthal opening angle between the muon transverse momentum direction and $\vec{p}_{T}^{\text {miss }}$. Another observable is the transverse momentum of final muon. In figures $4(\mathrm{a}, \mathrm{b}, \mathrm{c}, \mathrm{d})$, we provide the $\mathrm{LO}$ and QCD NLO corrected distributions of transverse momentum of muon from $W$ decay $\left(p_{T}^{\mu}\right)$, invariant transverse mass $M_{T}$ and the corresponding $K$-factors at the $8 \mathrm{TeV}$ and $14 \mathrm{TeV}$ LHC, respectively, where we take the mass of DM and coefficients $\Lambda$ as $m_{\chi}=10 \mathrm{GeV}$ and $\Lambda=1 \mathrm{TeV}$. Due to all the LO and NLO and $K$-factor cureves for D8 coupling are nearly overlapped with the corresponding ones for D5 coupling, we will don't distinguish between them in figures 4(a,b,c,d). From these figures, we can see that the QCD NLO correction obviously modifies the LO differential cross section. For the transverse momentum distributions of muon, the $K$-factors decrease slowly with the increment of $p_{T}^{\mu}$ from $25 \mathrm{GeV}$ to $250 \mathrm{GeV}$ for both $\sqrt{s}=8 \mathrm{TeV}$ and $\sqrt{s}=14 \mathrm{TeV}$ LHC. For the distributions of invariant transverse mass $M_{T}$, the QCD NLO corrected differential cross section is very different from the LO differential cross section at the lower and larger plotted $M_{T}$ regions. This is due to that the real gluon and real 

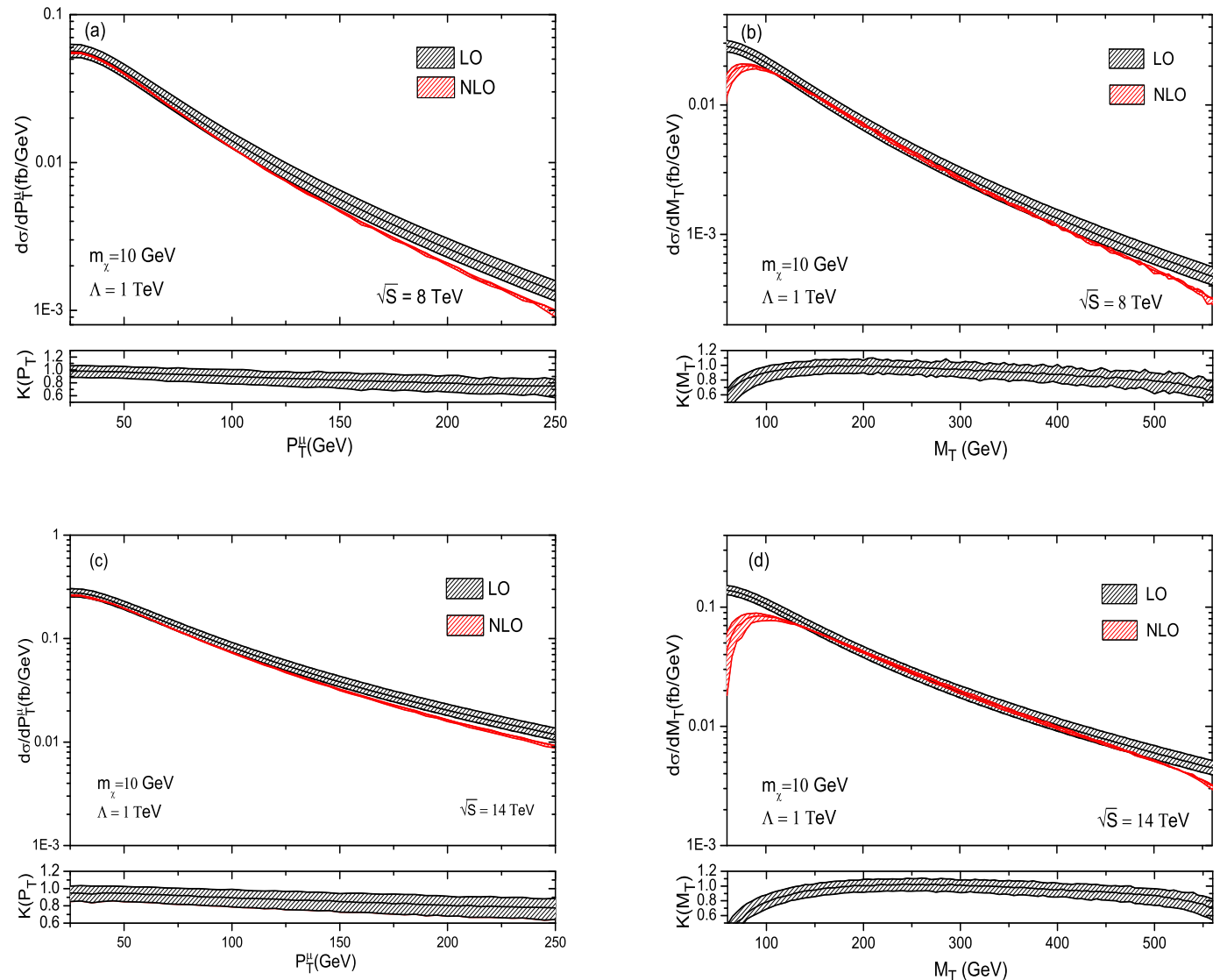

Figure 4. The LO and QCD NLO corrected distributions of the transverse momenta of the final muon, transverse mass $M_{T}$ and the corresponding $K$-factors for the $p p \rightarrow \bar{\chi} \chi W^{ \pm}+X \rightarrow \bar{\chi} \chi \mu^{ \pm} \stackrel{(-)}{\nu}_{\mu}$ $+X$ processes at the $\sqrt{s}=8 \mathrm{TeV}$ and $\sqrt{s}=14 \mathrm{TeV}$ LHC. The solid line indicates the differential cross section obtained with the default scale $\mu=\mu_{0}$, the shaded band represents the deviation from this scale when the scales are varied by a factor of two in each direction.

quark radiations have an additional jet in the final states, which changes the distributions of invariant transverse mass $M_{T}$.

\section{Summary}

The LHC provides an ideal facility to search for DM, offering complementary results to those obtained from direct detection experiments. Model independent searches for the pair production of DM require some other visible activity in the event, e.g. jets, photons, or vector bosons. In this paper we investigate the complete QCD NLO corrections to the $\bar{\chi} \chi W^{ \pm}$associated production at the LHC. We present the dependence of the LO and the QCD NLO corrected cross sections on the factorization/renormalization energy scale, and it shows that the dependence of the NLO cross section on the factorization/renormalization scale $\left(\mu=\mu_{f}=\mu_{r}\right)$ are significantly reduced. We present the LO and the QCD NLO 
corrected transverse momentum distributions of muon from $W$ boson decays $\left(p_{T}^{\mu}\right)$ and invariant transverse mass $M_{T}$. We find that the QCD NLO radiative corrections obviously modify the LO kinematic distributions, and the values of $K$-factor are obviously related to the phase space and the kinematic observables. It shows that we should consider the NLO QCD corrections in precision experimental data analysis for this process.

\section{Acknowledgments}

This work was supported in part by the National Natural Science Foundation of China (No.11205003, No.11305001, No.11275190, No.11375171, NO.11175001), the Key Research Foundation of Education Ministry of Anhui Province of China (No.KJ2012A021), the Youth Foundation of Anhui Province(No.1308085QA07), and financed by the 211 Project of Anhui University (No.02303319).

Open Access. This article is distributed under the terms of the Creative Commons Attribution License (CC-BY 4.0), which permits any use, distribution and reproduction in any medium, provided the original author(s) and source are credited.

\section{References}

[1] G. Bertone, D. Hooper and J. Silk, Particle dark matter: evidence, candidates and constraints, Phys. Rept. 405 (2005) 279 [hep-ph/0404175] [INSPIRE].

[2] J.L. Feng and J. Kumar, The WIMPless miracle: dark-matter particles without weak-scale masses or weak interactions, Phys. Rev. Lett. 101 (2008) 231301 [arXiv:0803.4196] [INSPIRE].

[3] J.L. Feng, H. Tu and H.-B. Yu, Thermal relics in hidden sectors, JCAP 10 (2008) 043 [arXiv:0808.2318] [INSPIRE].

[4] S.P. Martin, A supersymmetry primer, Adv. Ser. Direct. High Energy Phys. 21 (2010) 1 [hep-ph/9709356] [INSPIRE].

[5] M. Drees, R. Godbole and P. Roy, Theory and phenomenology of sparticles: an account of four-dimensional $N=1$ supersymmetry in high energy physics, World Scientific, Hackensack U.S.A. (2004) [INSPIRE].

[6] T. Appelquist, H.-C. Cheng and B.A. Dobrescu, Bounds on universal extra dimensions, Phys. Rev. D 64 (2001) 035002 [hep-ph/0012100] [INSPIRE].

[7] N. Arkani-Hamed, A.G. Cohen and H. Georgi, Electroweak symmetry breaking from dimensional deconstruction, Phys. Lett. B 513 (2001) 232 [hep-ph/0105239] [INSPIRE].

[8] DAMA and LIBRA collaborations, R. Bernabei et al., New results from DAMA/LIBRA, Eur. Phys. J. C 67 (2010) 39 [arXiv:1002.1028] [inSPIRE].

[9] CoGeNT collaboration, C.E. Aalseth et al., Results from a search for light-mass dark matter with a P-type point contact germanium detector, Phys. Rev. Lett. 106 (2011) 131301 [arXiv: 1002.4703] [INSPIRE].

[10] CDMS collaboration, J. Filippini, WIMP hunting with the cryogenic dark matter search, in Les Rencontres de Physique de la Valle d'Aosta, Italy (2009)

[Nuovo Cim. C 32N5-6 (2009) 45] [INSPIRE]. 
[11] XENON100 collaboration, E. Aprile et al., First dark matter results from the XENON100 experiment, Phys. Rev. Lett. 105 (2010) 131302 [arXiv: 1005.0380] [INSPIRE].

[12] P. Sorensen, A coherent understanding of low-energy nuclear recoils in liquid xenon, JCAP 09 (2010) 033 [arXiv: 1007.3549] [INSPIRE].

[13] A. Birkedal, K. Matchev and M. Perelstein, Dark matter at colliders: a model independent approach, Phys. Rev. D 70 (2004) 077701 [hep-ph/0403004] [INSPIRE].

[14] M. Beltrán, D. Hooper, E.W. Kolb and Z.C. Krusberg, Deducing the nature of dark matter from direct and indirect detection experiments in the absence of collider signatures of new physics, Phys. Rev. D 80 (2009) 043509 [arXiv: 0808.3384] [InSPIRE].

[15] Q.-H. Cao, C.-R. Chen, C.S. Li and H. Zhang, Effective dark matter model: relic density, CDMS II, Fermi LAT and LHC, JHEP 08 (2011) 018 [arXiv:0912.4511] [INSPIRE].

[16] M. Beltrán, D. Hooper, E.W. Kolb, Z.A.C. Krusberg and T.M.P. Tait, Maverick dark matter at colliders, JHEP 09 (2010) 037 [arXiv: 1002.4137] [INSPIRE].

[17] W. Shepherd, T.M.P. Tait and G. Zaharijas, Bound states of weakly interacting dark matter, Phys. Rev. D 79 (2009) 055022 [arXiv:0901.2125] [INSPIRE].

[18] CDF collaboration, T. Aaltonen et al., A search for dark matter in events with one jet and

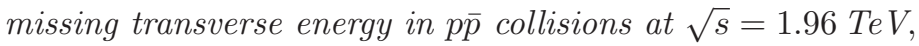

Phys. Rev. Lett. 108 (2012) 211804 [arXiv:1203.0742] [INSPIRE].

[19] ATLAS collaboration, Search for dark matter candidates and large extra dimensions in events with a jet and missing transverse momentum with the ATLAS detector, JHEP 04 (2013) 075 [arXiv:1210.4491] [INSPIRE].

[20] CMS collaboration, Search for dark matter and large extra dimensions in monojet events in pp collisions at $\sqrt{s}=7 \mathrm{TeV}$, JHEP 09 (2012) 094 [arXiv:1206.5663] [INSPIRE].

[21] ATLAS collaboration, Search for dark matter candidates and large extra dimensions in events with a photon and missing transverse momentum in pp collision data at $\sqrt{s}=7 \mathrm{TeV}$ with the ATLAS detector, Phys. Rev. Lett. 110 (2013) 011802 [arXiv:1209.4625] [INSPIRE].

[22] CMS collaboration, Search for dark matter and large extra dimensions in pp collisions yielding a photon and missing transverse energy, Phys. Rev. Lett. 108 (2012) 261803 [arXiv: 1204.0821] [INSPIRE].

[23] ATLAS collaboration, Search for dark matter pair production in events with a hadronically decaying $W$ or $Z$ boson and missing transverse momentum in pp collision data at $\sqrt{s}=8 \mathrm{TeV}$ with the ATLAS detector, ATLAS-CONF-2013-073, CERN, Geneva Switzerland (2013).

[24] CMS collaboration, Search for dark matter in the mono-lepton channel with pp collision events at center-of-mass energy of $8 \mathrm{TeV}$, CMS-PAS-EXO-13-004, CERN, Geneva Switzerland (2013).

[25] ATLAS collaboration, Search for dark matter in events with a hadronically decaying $W$ or $Z$ boson and missing transverse momentum in pp collisions at $\sqrt{s}=8$ TeV with the ATLAS detector, Phys. Rev. Lett. 112 (2014) 041802 [arXiv:1309.4017] [INSPIRE].

[26] U. Haisch, F. Kahlhoefer and J. Unwin, The impact of heavy-quark loops on LHC dark matter searches, JHEP 07 (2013) 125 [arXiv: 1208.4605] [INSPIRE].

[27] P.J. Fox and C. Williams, Next-to-leading order predictions for dark matter production at hadron colliders, Phys. Rev. D 87 (2013) 054030 [arXiv:1211.6390] [INSPIRE]. 
[28] F.P. Huang, C.S. Li, J. Wang and D.Y. Shao, Searching for the signal of dark matter and photon associated production at the LHC beyond leading order,

Phys. Rev. D 87 (2013) 094018 [arXiv:1210.0195] [INSPIRE].

[29] J. Wang, C.S. Li, D.Y. Shao and H. Zhang, Next-to-leading order QCD predictions for the signal of dark matter and photon associated production at the LHC,

Phys. Rev. D 84 (2011) 075011 [arXiv: 1107.2048] [INSPIRE].

[30] U. Haisch, F. Kahlhoefer and E. Re, QCD effects in mono-jet searches for dark matter, JHEP 12 (2013) 007 [arXiv:1310.4491] [INSPIRE].

[31] Y. Bai and T.M.P. Tait, Searches with mono-leptons, Phys. Lett. B 723 (2013) 384 [arXiv: 1208.4361] [INSPIRE].

[32] L.M. Carpenter, A. Nelson, C. Shimmin, T.M.P. Tait and D. Whiteson, Collider searches for dark matter in events with a $Z$ boson and missing energy, Phys. Rev. D 87 (2013) 074005 [arXiv: 1212.3352] [INSPIRE].

[33] N.F. Bell et al., Searching for dark matter at the LHC with a mono-Z, Phys. Rev. D 86 (2012) 096011 [arXiv: 1209.0231] [INSPIRE].

[34] J. Goodman et al., Constraints on light Majorana dark matter from colliders, Phys. Lett. B 695 (2011) 185 [arXiv:1005.1286] [INSPIRE].

[35] Y. Bai, P.J. Fox and R. Harnik, The Tevatron at the frontier of dark matter direct detection, JHEP 12 (2010) 048 [arXiv: 1005.3797] [InSPIRE].

[36] J. Goodman et al., Constraints on dark matter from colliders, Phys. Rev. D 82 (2010) 116010 [arXiv:1008.1783] [InSPIRE].

[37] A. Rajaraman, W. Shepherd, T.M.P. Tait and A.M. Wijangco, LHC bounds on interactions of dark matter, Phys. Rev. D 84 (2011) 095013 [arXiv:1108.1196] [INSPIRE].

[38] P.J. Fox, R. Harnik, J. Kopp and Y. Tsai, Missing energy signatures of dark matter at the LHC, Phys. Rev. D 85 (2012) 056011 [arXiv:1109.4398] [inSPIRE].

[39] K. Cheung, P.-Y. Tseng, Y.-L.S. Tsai and T.-C. Yuan, Global constraints on effective dark matter interactions: relic density, direct detection, indirect detection and collider, JCAP 05 (2012) 001 [arXiv: 1201.3402] [INSPIRE].

[40] G. Passarino and M.J.G. Veltman, One loop corrections for $e^{+} e^{-}$annihilation into $\mu^{+} \mu^{-}$in the Weinberg model, Nucl. Phys. B 160 (1979) 151 [InSPIRE].

[41] A. Denner and S. Dittmaier, Reduction schemes for one-loop tensor integrals, Nucl. Phys. B 734 (2006) 62 [hep-ph/0509141] [INSPIRE].

[42] A. Denner, Techniques for calculation of electroweak radiative corrections at the one loop level and results for $W$ physics at LEP-200, Fortsch. Phys. 41 (1993) 307 [arXiv:0709.1075] [INSPIRE].

[43] R.K. Ellis and G. Zanderighi, Scalar one-loop integrals for QCD, JHEP 02 (2008) 002 [arXiv:0712.1851] [INSPIRE].

[44] G. 't Hooft and M.J.G. Veltman, Scalar one loop integrals, Nucl. Phys. B 153 (1979) 365 [INSPIRE].

[45] A. Denner, U. Nierste and R. Scharf, A compact expression for the scalar one loop four point function, Nucl. Phys. B 367 (1991) 637 [INSPIRE]. 
[46] A. Denner and S. Dittmaier, Reduction of one loop tensor five point integrals, Nucl. Phys. B 658 (2003) 175 [hep-ph/0212259] [INSPIRE].

[47] B.W. Harris and J.F. Owens, The two cutoff phase space slicing method, Phys. Rev. D 65 (2002) 094032 [hep-ph/0102128] [INSPIRE].

[48] S. Mao et al., Next-to-leading order QCD corrections to $H W^{ \pm} \gamma$ production at the $L H C$, Phys. Rev. D 88 (2013) 076002 [arXiv:1310.0946] [inSPIRE].

[49] S. Mao et al., QCD corrections to associated Higgs boson production with a $W$ boson pair at the LHC, Phys. Rev. D 79 (2009) 054016 [arXiv: 0903.2885] [INSPIRE].

[50] J. Pumplin et al., New generation of parton distributions with uncertainties from global QCD analysis, JHEP 07 (2002) 012 [hep-ph/0201195] [INSPIRE].

[51] Particle Data Group collaboration, K. Nakamura et al., Review of particle physics, J. Phys. G 37 (2010) 075021 [inSPIRE]. 